\title{
An Improved Multi-Hop-Enabled Energy Efficient MAC Protocol for Underwater Acoustic Sensor Networks
}

\author{
Tomal Kumer Mozumder (MSc) \\ Sajjad Waheed (PhD)
}

Department of Information and Communication Technology

Mawlana Bhasani Science and Technology University, Bangladesh

doi: 10.19044/esj.2017.v13n15p240 URL:http://dx.doi.org/10.19044/esj.2017.v13n15p240

\begin{abstract}
Packet collisions occurred by hidden and local nodes in multi-hop enabled underwater acoustic sensor networks (UWASNs) have effect on throughput, energy efficiency and end-to-end delay. Existing Multi-HopEnabled Energy Efficient MAC Protocol for Underwater Acoustic Sensor Networks (MHEE MAC) utilized a double-phase contention resolution mechanism, which causes visit multiple time slot and energy overhead. In this paper, we propose a MAC protocol that use contention resolution mechanism with unique priority to provide energy efficiency. First, local nodes are eliminated comparing their priority and later, hidden nodes are mitigated. A simulation of proposed protocol is also developed to analyze the performance. Results obtained through simulation show that the proposed protocol achieves significantly lower energy consumption, reserve more energy and more stable throughput compared to MHEE-MAC, T-Lohi and slotted floor acquisition multiple access (S-FAMA).
\end{abstract}

Keywords: MAC protocols for underwater sensor networks (UWASNs); underwater communication; MAC protocol; sensor network; multi-hop network

\section{Introduction}

Two thirds of the earth surface is composed of water. There are still many un-explored areas in underwater compared with our land. This needs significant research efforts. Due to advanced underwater applications for commercial and military purposes, the research of UANs is increasing. In recent years more and more research interest and efforts are shifting to this area. The first underwater acoustic (UWA) communication was introduced during World War II (Rossing, 2007) (Khalil et al., 2017). Applications like 
exploration of underwater resources, forecasting and preventing natural and man-made disasters, monitoring environment of underwater, marine habitats, military and commercial surveillance (Akyildiz et al., 2006) (Partan et al., 2006) (Heidemann et al., 2006) (Stojanovic, 2003) (Sozer et al., 2000) are increasing day by day. To make it happen underwater communications needed with underwater devices. Underwater sensor nodes possess selfconfiguration capabilities. They perform their operation by exchanging location, configuration, and movement information and to send monitored data to an onshore receiver. Most systems have a large latency. Latency means the delay between the collection and the utilization of data. For the improvement of UWA communication, a standard similar to IEEE 802.11 is required (IEEE 802.11 Working Group, 1999). Medium access control (MAC) protocol is efficient and effective multi-hop networking for UWA networks.

UWA communication has many challenges like propagation for the low speed of the sound in the water $(1500 \mathrm{~m} / \mathrm{s})$ compared to the speed of radio waves $(3 * 108 \mathrm{~m} / \mathrm{s})$. The high propagation delay does not utilize CSMA (Syed et al., 2007). Moreover, UWA is highly non-symmetric. In UWA the transmission energy consumption is approximately one hundred times more than the reception energy consumption (Syed et al., 2008). Packet collisions reduce the channel utilization and consume precious energy resources. One recommendation is multi-hop mesh network technology (Proakis et al., 2001).

Two big challenges for a multi-hop underwater acoustic sensor network (UASN) are to minimize packet collisions (for both control and data) and to increase energy efficiency. Frame collisions in UASNs are occurred by local and hidden nodes. Channel reservation using control packets is one of the techniques. Therefore, T-Lohi were proposed (Syed et al., 2008). T-Lohi was designed for single-hop UWASN. In the proposal, contention resolution is performed by Beacon Frame and then, request to send (RTS) and clear to send (CTS). The unique priority of Beacon resolution reduces energy consumption, collision and delay in multi-hop UASNs.

In this paper, the proposed MAC protocol will be described; network simulations are performed to evaluate the proposed protocol compared to a prominent UWASN MAC protocol (MHEE-MAC). The rest of the paper include the related work in section 2. Our proposed protocol is described in Section 3. In Section 4, simulation results are demonstrated. Conclusions are provided in Section 5. 


\section{Related Work}

For the importance of energy efficiency an energy-efficient MAC protocol (EE-MAC) for highly-dense, short-range and fully-connected UASNs (Rodoplu and Park, 2005) was proposed, that has a very low duty cycle similar to the S-MAC (Ye et al., 2002). EE-MAC improves energy efficiency by minimizing the idle listening and reduces the energy loss due to packet collisions but is not suitable for multi-hop networks, because it does not have any collision avoidance mechanism which is important in multi-hop networks.

Due to the large propagation delay in UASN, slotted ALOHA (SALOHA) behaves similar to pure ALOHA with a guard time equal to the maximum propagation delay of the network (Syed et al., 2007). ALOHA was used in multi-hop UASN for string topology in (Gibson et al., 2007). The slotted floor acquisition multiple access (S-FAMA) protocol for UWASN was introduced in (Molins and Stojanovic, 2007). Later it extends the FAMA non-persistent carrier sensing (FAMA-NCS) protocol (Garcia-Luna-Aceves and Fullmer, 1999). It was for terrestrial wireless networks. In FAMA (Fullmer and Garcia-Luna-Aceves, 1995) the channel is acquired by a potential transmitting station prior to any transmission of data. This technique uses RTS and CTS. For the collision avoidance multiple access collision avoidance for wireless LAN (MACAW) (Bharghavan etal., 1994) was proposed. FAMA-NCS improved FAMA using long RTS and CTS control packets which ensure collision-free transmission of data in multi-hop networks with large propagation delay. S-FAMA reduces the need for long RTS and CTS. This is performed by slotting and synchronizing frames and constraining the transmission of control and data packets at the beginning of a slot which was required in FAMA-NCS. S-FAMA and MACAW protocols reduces collisions caused by local and hidden nodes through a single RTS/CTS cycle.

T-Lohi was proposed for UASN (Syed et al., 2008) which suggested the use of tones to reserve the channel. T-Lohi does not solve the hidden station problem and does not perform well in multi-hop networking. The micro-modem proposed by Woods Hole Oceanographic Institution (WHOI) (Lee et al., 2013) is a small-footprint, low-power acoustic modem and has capability to be used as a low power tone receiver. It has also the capability to be used as a data receiver.

TDMA-based MAC protocol, efficient communication scheduling (ECS) (Hong et al., 2011) with continuous time slicing was proposed which shows improvement in channel utilization. A multi-hop reservation MAC protocol (Lee and Cho, 2014) was proposed in which a single reservation was used for a multi-hop multiple packet transmission. It provides a better end-to-end delay and throughput for uni-directional data dissemination. But 
experiences longer reservation time data. A delay-aware MAC protocol algorithm (Noh et al., 2010) was proposed in which the neighboring nodes are used to calculate propagation delays. It uses the time-stamp information embedded in RTS packets. Secure Cooperation of Autonomous Mobile Sensors Using an Underwater Acoustic Network (Caiti et al., 2012) described a methodology for secure cooperation within a network of autonomous mobile underwater sensors connected through an acoustic communication network. A cooperative algorithm based on the behavioral paradigm has been illustrated. Underwater Acoustic Wireless Sensor Networks: Advances and Future Trends in Physical, MAC and Routing Layers (Climent et al., 2014) presents a comprehensive view of the current state-of-the-art in UWSNs by analyzing the current research status of the physical, MAC and routing layers. RF Path and Absorption Loss Estimation for Underwater Wireless Sensor Networks in Different Water Environments (Qureshi et al., 2016) highlights the characteristics of the channel and possible effects over the EM frequencies, specifically over the $2.4 \mathrm{GHz}$ ISM frequency band. A Survey on Underwater Acoustic Sensor Networks: Perspectives on Protocol Design for Signaling, MAC and Routing (SharifYazd et al., 2017) contains PHY layer constraint, MAC and routing design, and new topics regarding signal processing of UASNs. Multi-Hop-Enabled Energy Efficient MAC Protocol (MHEE-MAC) (Shazzad et al., 2015) for Underwater Acoustic Sensor Networks was an improvement over T-Lohi and S-FAMA protocols.

\section{Proposed Protocol}

Assume a typical multi-hop network as shown in Figure 1. Two node sets, $\mathrm{s}=\{\mathrm{S} 0, \mathrm{~S} 1, \mathrm{~S} 2\}$ and $\mathrm{h}=\{\mathrm{H} 0, \mathrm{H} 1, \mathrm{H} 2\}$ are considered out of interference range. They are in the range of a possible receiving node, $r=$ $\{R\}$. Here, the source node member of $s$ and $h$ sets. And $r$ is the receiver.

Any node set $\mathrm{Si}, \mathrm{Sj} € \mathrm{~s}$ and $\mathrm{Hk}, \mathrm{Hl} € \mathrm{~h}$ are out of the interference range (where $\mathrm{i} \neq \mathrm{j}$ and $\mathrm{k} \neq 1$ ). The nodes are connected to each other by only a single node. The node sets ( $\mathrm{s}$ and $\mathrm{h}$ ) are in local collision domains. Each node in $\mathrm{s}$ and $\mathrm{h}$ sets can contend for the channel independently with each other using a random unique priority number $0-n$ (nodes) assigned to each node. After first time slot, a single node from each set would have won the contention. And then reserved the channel to send a data packet to $R$. The nodes $\mathrm{S} 0$ and $\mathrm{H} 0$ do not have any information about the state of the other. Because they are hidden to each other. If they send a data packet to $\mathrm{R}$, they (two packets) arrive at the same time, which may cause a collision. Each node in the sets $\mathrm{s}$ and $\mathrm{h}$ are in a hidden collision domain. 


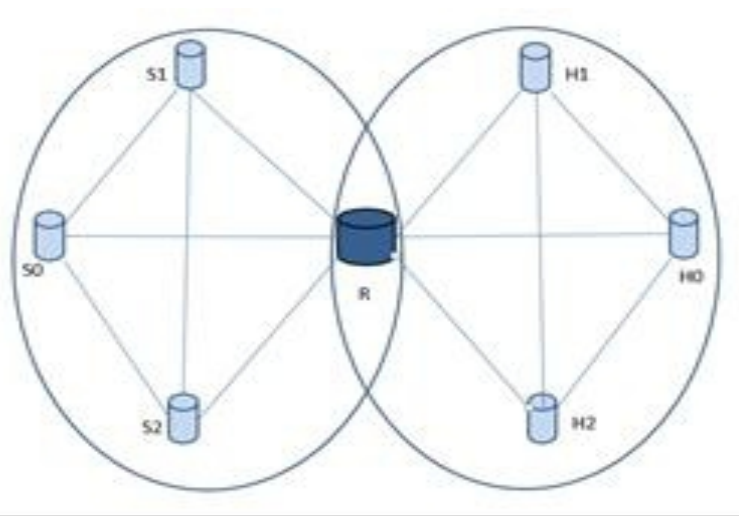

Figure 3.1. Network topology based on interference range.

Collision avoidance in a multi-hop network depends on two distinct collision domains, i.e., local collision domain and hidden collision domain. Our proposal is to separate the channel contention into two phases. In Phase 1 , data packet collisions are avoided through local channel reservation (local domain). In Phase 2, data packet collisions are avoided through controlling the corresponding hidden channels (hidden collision domain).

\section{Protocol Description}

The successful transmission and reception of data is operated by the use of a logical time frame (variable length). A time frame includes multiple time slots. In our proposed MAC protocol, both phases consist of one or more time slots. Every slot is called a contention round (CR). In first time slot each node will send a Beacon frame with unique priority [0-n (nodes)].The node with highest priority from different collision domain transmit a RTS in second time slot. The receiver response with a CTS packet which transmit its RTS at latest time. The duration of the Beacon Frame (BF) is determined such that any transmitted control packet must be received by all nodes (Syed et al., 2008) (Molins and Stojanovic, 2007) (Garcia-LunaAceves and Fullmer, 1999). Energy lost due to idle listening, generally two different types of receivers are used (1) a low power wake-up tone receiver (Lee et al., 2013) and (2) a data receiver. For energy saving the data receiver is kept in sleep mode most of the time. When needed, the data receiver is turned on by the wake-up tone receiver. Therefore, a data packet always follows a wake-up Beacon. All nodes in the network are assumed to be synchronized. The transmission of each node must start at the beginning of a slot. The maximum propagation delay must be equal to the minimum duration of a slot between any pair of nodes in the network plus the time duration required to detect any wake-up Beacon by the receiver. 


\begin{tabular}{|c|c|c|c|c|c|c|c|c|}
\hline $\begin{array}{c}\text { HW } \\
\text { Preamble }\end{array}$ & $\begin{array}{c}\text { Frame } \\
\text { Length }\end{array}$ & FCF & SRC & WB & DST & $\begin{array}{c}\text { Random } \\
\text { Priority }\end{array}$ & $\begin{array}{c}\text { Msg } \\
\text { ID }\end{array}$ & FCS \\
\hline
\end{tabular}

Figure 3.2. Beacon frame structure for the proposed protocol.

In the local link reservation phase, short Beacons with unique priority are used to resolve contention among the nodes. At the beginning of a frame, the node starts contending for the channel by transmitting a beacon. At beacon frame the random priority are incorporated. The all receiving nodes among neighbor contending nodes compare the random priority with its own priority. If any contending nodes ensure that the received short beacons random priority is greater than the own self random priority then the nodes goes to sleep for three time slot. This completes a single contention round. In this period, if the contending node does not receive any short beacon, the local link reservation is successful. And the hidden link control phase can be started. This local link reservation phase is similar to the channel reservation technique used in (Syed et al., 2008). In local link reservation, noncontending node that received a tone while in back-off state enters into a quiet state.

To eliminate collisions in hidden collision domains, the local link reservation is supported by the hidden link control phase. Hidden link control is performed by the exchange of two distinct control packets before a data transmission. The additional control packets are RTS and CTS. The sender broadcasts RTS and waits for one more slot to receive the CTS from the receiver after the local link reservation. It sends its data packet in the next slot if the sender successfully receives a CTS from the receiver. Thus collisions are avoided. It backs off and invalidates its local link reservation if the sender does not receive CTS from the receiver within the next slot duration. Nodes within the interference range of the sender receive an RTS packet and go to sleep mode until the end of the next slot, thus conserving energy. The nodes within the interference range of the destination receive CTS and also go to sleep mode until the end of the transmission between the sender and the receiver. In a given slot a node receiving more than one successful RTS packet prioritizes those packets and selects one node to be the potential sender. This is performed by putting the destination field address as the potential sender's address. 


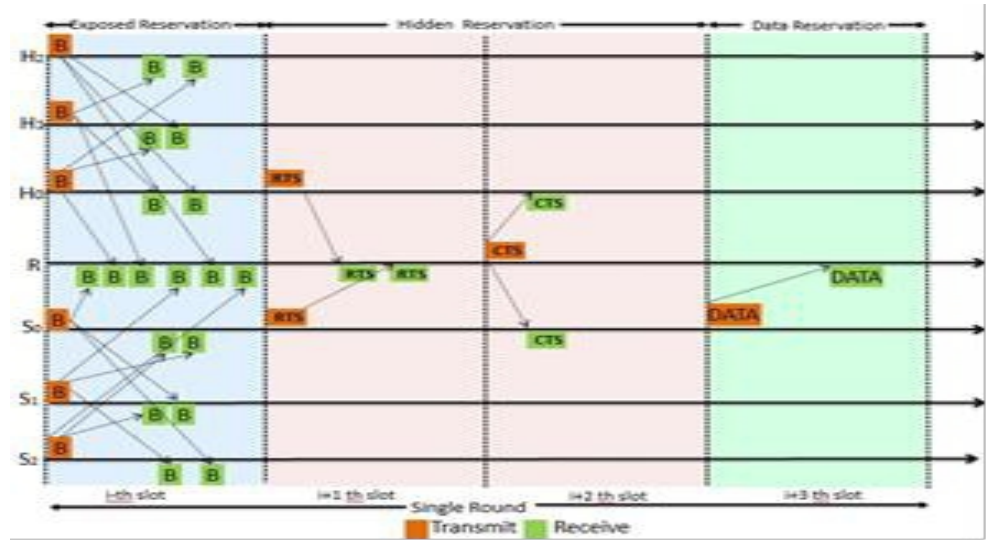

Figure 3.3.The proposed protocol timing diagram.

A typical timing diagram (which is represented by a logical time frame) scenario is depicted in Figure 3 for a typical network scenario shown in Figure 1. Consider that each node of the collision domains wants to send the data packet to the receiving node R. Nodes S0, S1 and S2 of the collision domain s and nodes $\mathrm{H} 0, \mathrm{H} 1$ and $\mathrm{H} 2$ of the collision domain h start contention for local link reservation by transmitting short beacon at the beginning of a frame (i-th slot). After transmission of the beacon, each contending node switches to listening mode to detect the beacon from neighbors. By the end of the slot, S0, S1 and S2 nodes would have recorded the number of contending nodes (number Of Contending Nodes), which is three in this scenario. Similarly, contention among H0, $\mathrm{H} 1$ and $\mathrm{H} 2$ is resolved by beacon, also, since each contending node from both collision domains experiences more than one contender, the nodes which receive higher random priority beacon switches its state to the back-off mode and the duration of the backoff mode is equal to three mini timing slots shown in timing diagram. Assume that nodes $\mathrm{S} 0$ and $\mathrm{H} 0$ win the channel due to their higher priority beacons, respectively. Therefore, S0 and $\mathrm{H} 0$ send RTS at the beginning of (i +1 )-th slot. The destination node, $\mathrm{R}$, receives both control packets successfully due to the slight delay caused by the uncertainty of the acoustic channel. Based on their arrival time the destination node $\mathrm{R}$ prioritizes the control packets and gives higher priority to the packet that arrives at the latest. Later, it broadcasts a CTS packet at the beginning of the $(i+2)$-th slot. While the transmission of the data packet continues from node $\mathrm{S} 0$ to node $\mathrm{R}$ all nodes of the domain $h$ keep themselves in sleep mode. Thus, by incorporating a two-phase reservation, collisions of data packets are avoided in multi-hop networks. 


\section{Algorithms for Transmitter Nodes According to Proposed MAC Protocol}

Algorithm 1: Exposed and hidden link reservation

Input: random_priority[number of nodes], distance[10], nodeId[number of nodes], win_channel, slot_time, sleep_time, range_radious;

Output: true, false //exposed link reservation

//each sender node decided either it win the channel or not

For i 1 to number of exposed nodes do

For $\mathrm{k} 1$ to number of exposed nodes do

If $(\mathrm{i} !=\mathrm{k} \& \&$ random_priority[i] $>$ random_priority[k])

win=random_priority $[\mathrm{i}]$;

else

win=random_priority $[\mathrm{k}]$;

sleep_time $=3 *$ slot_time; // lower priority nodes goes to sleep

end for I and $\mathrm{k}$

// Hidden link Reservation

For I 1 to number of exposed nodes do

If $($ win $==$ nodeId $[i])$

Win_channel=true;

Broadcast (RTS_packet);

Else

Win_channel=false;

End for

Void Broadcast (cPacket * RTS_packet)

\{

For I 1 to $\mathrm{n}$ do

If(range_radious $<=$ distance $[i])$

Send RTS packet to ith node including receiver \}

Algorithm 2: Receiver CTS Transmission and Data packet Reception

Input: transmit_time, p_d[nodeId], duration, arrival_time[10], random_priority[10], Sleep_time, slot_time, CTS_sender=1000;

Output: CTS_sender ;

For i 1 to number of RTS packet do

For $\mathrm{k} 1$ to number of RTS packet do

If(i != k \&\& arrival_time[i] > arrival_time[k])

CTS_sender[i] ;

Else

CTS_sender[k] ;

Sleep_time=slot_time; 
End for I and $\mathrm{k}$

If(CTS_sender $==1000)$

// receiver confirms that RTS packet of $\mathrm{x}$ and collide with each other

Int Capture_effect( X RTS_packet, y RTS_packet )

If(random_priority[x]> random_priority[y])

\{ CTS_sender $=x$;

Else

CTS_sender=y;

\}

Int capture_effect( X cPAcket* RTS packet, y cPAcket * RTS packet)

\{

If $\left(\right.$ transmit_time $[\mathrm{x}]+\mathrm{p} \_\mathrm{d}[\mathrm{x}]<$ transmit_time $[\mathrm{y}]+\mathrm{p} \_\mathrm{d}[\mathrm{y}] \& \&$

transmit_time[x]+p_d[x]+duration $>$ transmit_time[y]+p_d[y]+duration)

// this condition certify that $\mathrm{x}$ and $\mathrm{y}$ 's RTS collide

Return

Random_ priority $\mathrm{x}$ and $\mathrm{y}$;

\}

\section{Simulation Results and Decision}

In this section, both the proposed protocol (IMHEE) and the MHEE protocol are simulated under similar conditions to demonstrate the effects of the improvement achieved with the proposed protocol. We used OMNET++ (Varga and Hornig, 2008) as the network simulation platform. We also used modified the MiXiM (Köpke et al., 2008) (Wessel et al., 2009) framework to make it suitable for UASNs. MiXiM is an OMNeT++ modelling framework which created for mobile and fixed wireless (Arellano and Mahgoub, 2013). We use $0.0010 \mathrm{sec}$ for each TDMA slot in simulation and our network area is $1000 \times 800 \mathrm{~m}$. We simulate the program for $6,10,20,30,40,50$ nodes and calculate the average result for $100 \mathrm{sec}$ simulation time.

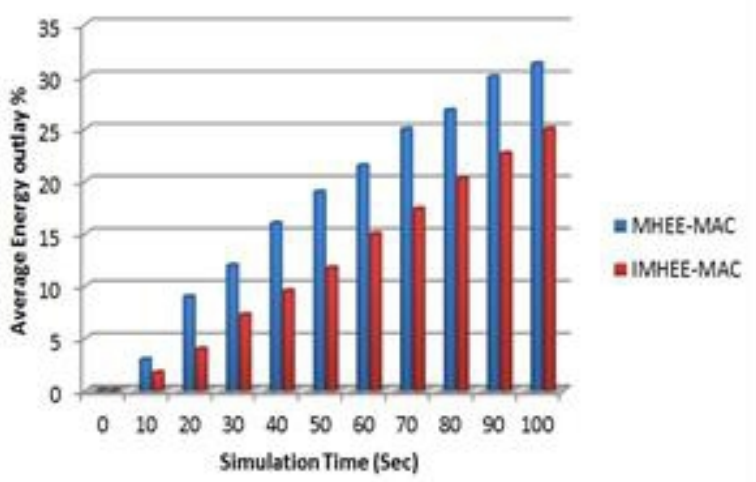

Figure 4.1. Average energy consumption with varying simulation time 
Due to the large number of participating nodes in the network, it can be considered as an advantage the short distance between them, using a multi-hop communication to save energy in data transmission.

In figure 4.1, shows the average energy consumption compared with the existing MAC protocol (MHEE-MAC). Energy consumption is higher for MHEE-MAC because each node has to active continuously till the full round. If we can keep only sending node active and all other nodes sleep, the data consumption will be low. According to our MAC technique, nodes are sleep and wake up according to their priority basis in first time slot and only prioritized node will send RTS. So the number of active nodes are decreasing in our system. As a result energy consumption is low.

Similarly, Figure 4.2 shows the higher network life time. As large number of nodes go to sleep mode according to their low priority, the network life span expands. So the energy reservation in our system is high compared to MHEE-MAC.

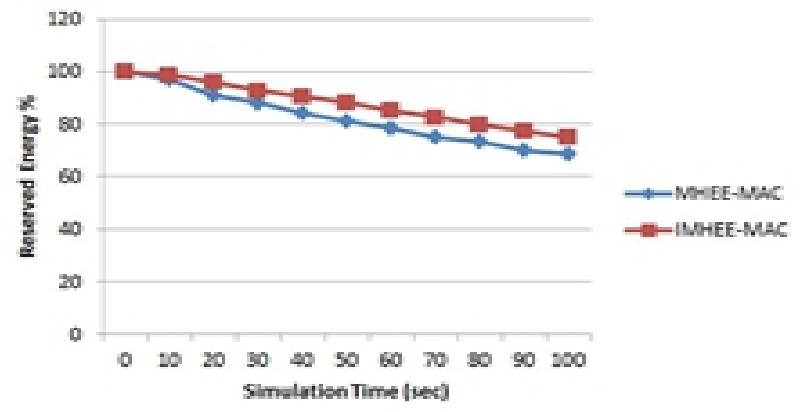

Figure 4.2. Reserved energy rate comparison with varying simulation time.

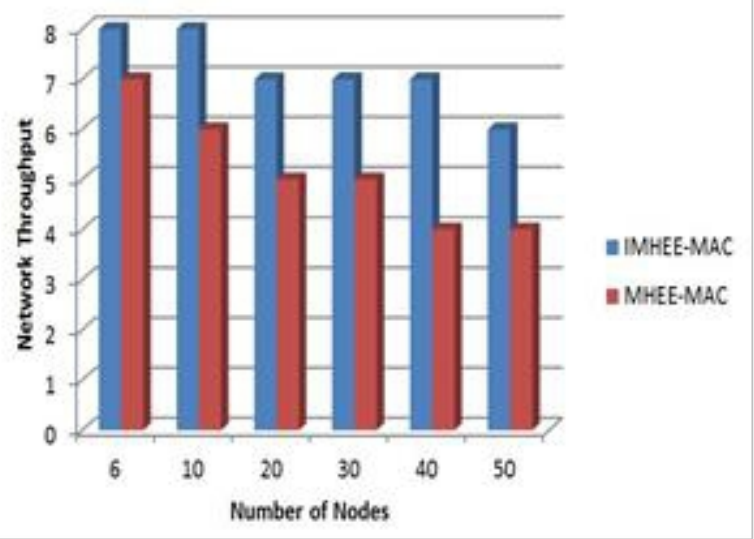

Figure 4.3. Network throughput measurement with varying number of nodes

Figure 4.3, represents the network improved throughput. Network throughput is increased in IMHEE-MAC due to less contention cycle. We need less number of contention cycle in IMHEE-MAC because of TDMA technique and improved routing based on random priority. 


\section{Conclusion}

A multi-hop-enabled energy efficient MAC protocol for UWASN has been proposed in this paper, which introduces a novel TDMA resolution technique with unique priority. The proposed protocol has been evaluated through simulations. The proposed protocol provides lower energy consumption, higher energy reservation and higher energy throughput compared to MHEE-MAC, T-Lohi and S-FAMA protocols.

\section{References:}

1. Rossing, T. (2007). Springer handbook of acoustics. New York, N.Y: Springer.

2. Khalil, M. I., Hossain, M. A., Mamtaz, R., Ahmed, I., \& Akter, M. (2017, February). Time Efficient Receiver Oriented Sleep Scheduling for Underwater Sensor Network. In Imaging, Vision \& Pattern Recognition (icIVPR), 2017 IEEE International Conference on (pp. 1-6). IEEE.

3. Akyildiz, I. F., Pompili, D., \& Melodia, T. (2006, September). Stateof-the-art in protocol research for underwater acoustic sensor networks. In Proceedings of the 1st ACM international workshop on Underwater networks (pp. 7-16). ACM.

4. Partan, J., Kurose, J., \& Levine, B. N. (2006). A survey of practical issues in underwater networks, international conference on mobile computing and networking. In Proc. of the 1st ACM international workshop on Underwater networks (pp. 17-24).

5. Heidemann, J., Ye, W., Wills, J., Syed, A., \& Li, Y. (2006, April). Research challenges and applications for underwater sensor networking. In Wireless Communications and Networking Conference, 2006. WCNC 2006. IEEE (Vol. 1, pp. 228-235). IEEE.

6. Stojanovic, M. (2003). Acoustic (underwater) communications. Encyclopedia of Telecommunications.

7. Sozer, E. M., Stojanovic, M., \& Proakis, J. G. (2000). Underwater acoustic networks. IEEE journal of oceanic engineering, 25(1), 7283.

8. IEEE 802.11 Working Group. (1999). Part11: Wireless LAN medium access control (MAC) and physical layer (PHY) specifications. ANSI/IEEE Std. 802.11.

9. Syed, A. A., Ye, W., Heidemann, J., \& Krishnamachari, B. (2007, September). Understanding spatio-temporal uncertainty in medium access with ALOHA protocols. In Proceedings of the second workshop on Underwater networks (pp. 41-48). ACM.

10. Syed, A. A., Ye, W., \& Heidemann, J. (2008, April). T-Lohi: A new class of MAC protocols for underwater acoustic sensor networks. In 
INFOCOM 2008. The 27th Conference on Computer Communications. IEEE (pp. 231-235). IEEE.

11. Proakis, J. G., Sozer, E. M., Rice, J. A., \& Stojanovic, M. (2001). Shallow water acoustic networks. IEEE communications magazine, 39(11), 114-119.

12. Rodoplu, V., \& Park, M. K. (2005, September). An energy-efficient MAC protocol for underwater wireless acoustic networks. In OCEANS, 2005. Proceedings of MTS/IEEE (pp. 1198-1203). IEEE.

13. Ye, W., Heidemann, J., \& Estrin, D. (2002). An energy-efficient MAC protocol for wireless sensor networks. In INFOCOM 2002. Twenty-first annual joint conference of the IEEE computer and communications societies. Proceedings. IEEE (Vol. 3, pp. 15671576). IEEE.

14. Gibson, J. H., Xie, G. G., Xiao, Y., \& Chen, H. (2007, June). Analyzing the performance of multi-hop underwater acoustic sensor networks. In OCEANS 2007-Europe (pp. 1-6). IEEE.

15. Molins, M., \& Stojanovic, M. (2007, May). Slotted FAMA: a MAC protocol for underwater acoustic networks. In OCEANS 2006-Asia Pacific (pp. 1-7). IEEE.

16. Garcia-Luna-Aceves, J. J., \& Fullmer, C. L. (1999). Floor acquisition multiple access (FAMA) in single-channel wireless networks. Mobile Networks and Applications, 4(3), 157-174.

17. Fullmer, C. L., \& Garcia-Luna-Aceves, J. J. (1995, October). Floor acquisition multiple access (FAMA) for packet-radio networks. In ACM SIGCOMM computer communication review (Vol. 25, No. 4, pp. 262-273). ACM.

18. Bharghavan, V., Demers, A., Shenker, S., \& Zhang, L. (1994). MACAW: a media access protocol for wireless LAN's. ACM SIGCOMM Computer Communication Review, 24(4), 212-225.

19. Lee, J. Y., Yun, N. Y., Muminov, S., Shin, S. Y., Ryuh, Y. S., \& Park, S. H. (2013). A focus on practical assessment of MAC protocols for underwater acoustic communication with regard to network architecture. IETE Technical Review, 30(5), 375-381.

20. Hong, L., Hong, F., Guo, Z., \& Li, Z. (2011). ECS: Efficient communication scheduling for underwater sensor networks. Sensors, 11(3), 2920-2938.

21. Lee, J. W., \& Cho, H. S. (2014). Cascading multi-hop reservation and transmission in underwater acoustic sensor networks. Sensors, 14(10), 18390-18409.

22. Noh, Y., Wang, P., Lee, U., Torres, D., \& Gerla, M. (2010, October). DOTS: A propagation delay-aware opportunistic MAC protocol for 
underwater sensor networks. In Network Protocols (ICNP), 2010 18th IEEE International Conference on (pp. 183-192). IEEE.

23. Varga, A., \& Hornig, R. (2008, March). An overview of the OMNeT++ simulation environment. In Proceedings of the 1 st international conference on Simulation tools and techniques for communications, networks and systems \& workshops (p. 60). ICST (Institute for Computer Sciences, Social-Informatics and Telecommunications Engineering).

24. Köpke, A., Swigulski, M., Wessel, K., Willkomm, D., Haneveld, P. T., Parker, T. E., ... \& Valentin, S. (2008, March). Simulating wireless and mobile networks in OMNeT++ the MiXiM vision. In Proceedings of the 1 st international conference on Simulation tools and techniques for communications, networks and systems \& workshops (p. 71). ICST (Institute for Computer Sciences, SocialInformatics and Telecommunications Engineering).

25. Wessel, K., Swigulski, M., Köpke, A., \& Willkomm, D. (2009, March). Mixim: the physical layer an architecture overview. In Proceedings of the 2nd International Conference on Simulation Tools and Techniques (p. 78). ICST (Institute for Computer Sciences, Social-Informatics and Telecommunications Engineering).

26. Arellano, W., \& Mahgoub, I. (2013, December). TrafficModeler extensions: A case for rapid VANET simulation using, OMNET++, SUMO, and VEINS. In High Capacity Optical Networks and Enabling Technologies (HONET-CNS), 2013 10th International Conference on (pp. 109-115). IEEE.

27. Caiti, A., Calabro, V., Dini, G., Lo Duca, A., \& Munafo, A. (2012). Secure cooperation of autonomous mobile sensors using an underwater acoustic network. Sensors, 12(2), 1967-1989.

28. Climent, S., Sanchez, A., Capella, J. V., Meratnia, N., \& Serrano, J. J. (2014). Underwater acoustic wireless sensor networks: advances and future trends in physical, MAC and routing layers. Sensors, 14(1), 795-833.

29. Qureshi, U. M., Shaikh, F. K., Aziz, Z., Shah, S. M. Z. S., Sheikh, A. A., Felemban, E., \& Qaisar, S. B. (2016). RF Path and Absorption Loss Estimation for Underwater Wireless Sensor Networks in Different Water Environments. Sensors, 16(6), 890.

30. Sharif-Yazd, M., Khosravi, M. R., \& Moghimi, M. K. (2017). A Survey on Underwater Acoustic Sensor Networks: Perspectives on Protocol Design for Signaling, MAC and Routing. arXiv preprint arXiv:1703.08353.

31. Shazzad, K., Tepe, K., \& Abdel-Raheem, E. (2015). Multi-HopEnabled Energy-Efficient MAC Protocol for Underwater Acoustic 
Sensor Networks. Journal of Sensor and Actuator Networks, 4(3), 226-250. 